\title{
Muon revolution frequency distribution from a partial-time Fourier transform of the g-2 signal in the muon g-2 experiment
}

\author{
Yuri Orlov $^{\mathrm{a}, *}$, Cenap S. Ozben ${ }^{\mathrm{b}}$, Yannis K. Semertzidis ${ }^{\mathrm{b}}$ \\ ${ }^{a}$ Newman Laboratory of Nuclear Studies, Cornell University, Ithaca, NY 14853-5001, USA \\ ${ }^{\mathrm{b}}$ Brookhaven National Laboratory, Department of Physics, Upton, NY 11973, USA
}

Received 5 April 2001; received in revised form 10 July 2001; accepted 12 July 2001

\begin{abstract}
A new method of precise measurement of the revolution frequency distribution, $F(f)$, in the muon storage ring, and hence muon momentum $p$, energy $E$, and equilibrium radius $R$ distributions, has been developed and used in analyzing data in the muon g-2 experiment at Brookhaven National Laboratory. The method is partly based on the Fourier transform of the observed electron decay signal, which is known in this experiment only after some time $t_{\mathrm{s}}$ after injection. It is shown that the standard Fourier transform would give a wrong frequency distribution even if the signal were known immediately after injection. Only the cosine Fourier transform with the properly determined initial time $t_{0}$ (different for different detectors placed along the orbit) gives the correct frequency distribution in such a case. As for a later starting time, $t_{\mathrm{s}}>t_{0}$, a special procedure must be used to find $t_{0}$ and to compensate for the lack of information about the signal between $t_{0}$ and $t_{\mathrm{s}}$. The new technique is highly accurate and radically different from that used by CERN in its muon g-2 experiment. (C) 2002 Elsevier Science B.V. All rights reserved.
\end{abstract}

PACS: $29.20 . \mathrm{Dh} ; 29.27 . \mathrm{Fh} ; 02.30 . \mathrm{Nw}$

Keywords: Storage ring; Momentum distribution; Fourier

\section{Introduction}

In the muon g-2 experiment [1], the injected muons with momenta distributed around the designed $P_{\mathrm{m}}=3.0944 \mathrm{GeV} / c$ (the so-called magic momentum) are rotating in the homogeneous magnetic field $B=1.4513 \mathrm{~T}$, in the ring of the magic radius $R_{\mathrm{m}}=7.112 \mathrm{~m}$. Electric quadrupoles provide the needed vertical focusing. The muon

*Corresponding author. Tel.: + 1-607-255-3502; fax: + 1607-254-4552.

E-mail address: orlov@mail.Ins.cornell.edu (Y. Orlov). lifetime $\tau=64.4 \mu \mathrm{s}$. During the muon rotations, their spins are precessing around their orbits with the $g-2$ frequency $\omega_{a}=a(e B / m c), a=(g-2) / 2$. In correlation with these spin precessions, the decay electron signal, $S(t)$, observed by the 24 detectors placed along the ring, oscillates with the same frequency. The ideal signal should have the form

$$
S(t)=N \mathrm{e}^{-t / \tau}\left[1+A \cos \left(\omega_{a} t+\phi\right)\right] .
$$

In the presence of the focusing electric field, $\vec{E}$, the more accurate formula for the spin precession 
frequency is

$\vec{\omega}_{a}=-\frac{e}{m c}\left[a \vec{B}-\left(a-\frac{1}{\gamma^{2}-1}\right) \vec{\beta} \times \vec{E}\right]$

$\beta=v / c$.

The choice of the magic muon energy with energyto-mass ratio $\gamma_{\mathrm{m}}=29.3,1 /\left(\gamma_{\mathrm{m}}^{2}-1\right)=a$, was made to eliminate the influence of the electric field on the $\omega_{a}$ value.

However, the distribution of muon $\gamma$-values, and hence momenta $p$, revolution frequencies $f$, and equilibrium radii $R$, around their magic values leads to a systematic error $\Delta \omega_{a} / \omega_{a}$ caused by a non-zero electric field $\vec{E}$ along the non-ideal muon orbit $[2,3]$ :

$$
\begin{aligned}
& \frac{\Delta \omega_{a}}{\omega_{a}}=-2 n(1-n)\left\langle\left(\frac{\Delta f}{f_{\mathrm{m}}}\right)^{2}\right\rangle, \Delta f=f-f_{\mathrm{m}}, \\
& n=\frac{R \partial E_{R} / \partial R}{\beta B} .
\end{aligned}
$$

Here $f_{\mathrm{m}}$ is the magic revolution frequency. Brackets $\langle x\rangle$ mean $x$ averaged over the distribution of the muon revolution frequencies. An example of the equilibrium radius distribution of muons measured (1999 run) by the method described in this paper is shown in Fig. 1. This method is radically different from the method used in CERN [3].

Turning to our technique, we should note that, in order to extract the muon frequency distribu-

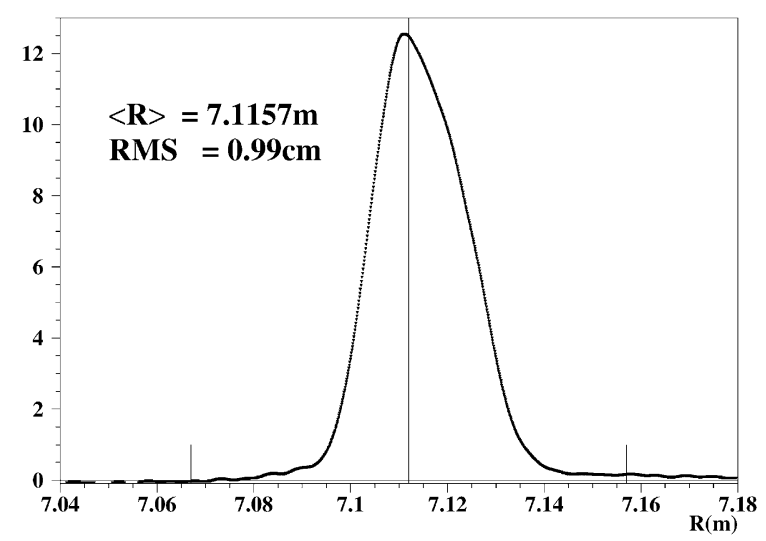

Fig. 1. An example of the equilibrium radii distribution. tion from the decay electron signal, we need to Fourier analyze not that signal itself, but the socalled fast rotation signal, $F(t)$, which is defined as the ratio of the actually observed signal to the fit function describing this signal, see Figs. 2a-c (simulations). In the case of the five parameters fit, we therefore have to analyze

$F(t)=\frac{S(t)}{N \mathrm{e}^{-t / \tau}\left[1+A \cos \left(\omega_{a} t+\phi\right)\right]}$.

The advantage of using this ratio is that both decay exponential, $\exp (-t / \tau)$, and the $\mathrm{g}-2$ frequency, $\omega_{a}$, are obviously excluded from $F(t)$ and hence do not disturb the Fourier spectrum of the revolution frequencies. Physically, $F(t)$ describes a signal which we would see if muons were stable spinless particles. $F(t)$ is the "rectified" signal whose magnitude does not go down exponentially; instead, the statistical error of any individual event in this signal grows exponentially in time. Note that since our muon bunches are initially short, the signal $F(t)$ includes not only a constant part of the muon current, and oscillations corresponding to the muon revolution frequencies, but also higher modes, $k>1$, of these frequencies.

\section{Some properties of partial-time Fourier analyses}

Any physical process we want to analyze lasts some finite time. If oscillations of a physical system are classical like the oscillations of the muon polarization averaged over quantum states of different muons, or, say, the rotations of these muons in the g-2 ring, then the finite time of oscillations does not forbid us to measure their frequencies with however high accuracy (limited only by statistical and systematic errors) by using some non-Fourier methods. But the accuracy of the Fourier transform is degraded by a finite time of observation, $\Delta t$. The reason is not merely the classical uncertainty $\Delta f$ of frequency $f$ found from the Fourier transform, $\Delta f \sim 1 /(\pi \Delta t)$. The accuracy is degraded even when a signal duration $\Delta t$ is sufficiently large, as, for example, in the g-2 case, so the uncertainty $\Delta f$ corresponding to this duration is small. The main phenomenon deforming the frequency distribution described by the 

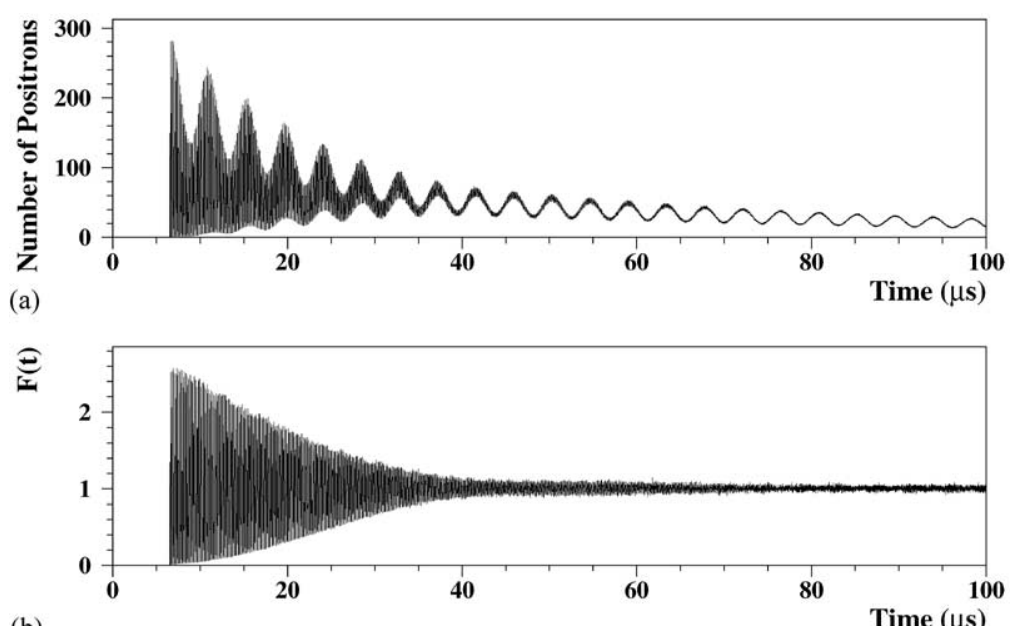

(b)

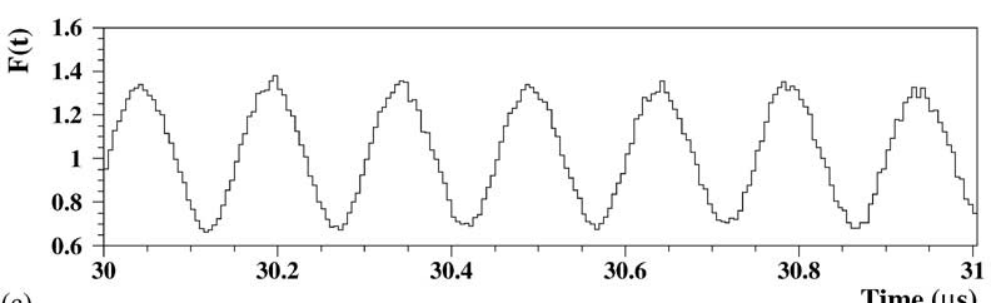

(c)

Time $(\mu s)$

Fig. 2. (a) Simulated decay electron signal at early times, $S(t)$. (b), (c) Fast rotation signal, $F(t)$.

Fourier transform is caused by the abrupt beginning of the observed signal at time $t_{0}$ or later. This deformation may not disappear even if the end time $t_{\mathrm{m}}$ of the signal goes to infinity, $\Delta t \equiv\left(t_{\mathrm{m}}-\right.$ $\left.t_{0}\right) \rightarrow \infty$. We will show this problem (and its solution) in a simple, analytically solvable example that is not so far from the actual situation in the g2 experiment.

We consider a very short initial muon bunch, $\Delta t_{\mathrm{B}} \rightarrow 0$, and will take into account that in such a case, the time dependence of the fast rotation signal, $F(t)$, observed at a given detector in the g-2 experiment, can be described as

$F(t)=\int \mathrm{d} f A F(f) \cos \omega\left(t-t_{0}\right), \quad t>t_{0}$

with $A \rightarrow 1$ when $\Delta t_{\mathrm{B}} \rightarrow 0$. In Eq. (5), $F(f) \mathrm{d} f$ is proportional to the initial number of muons having revolution frequency $\omega=2 \pi f$. The spectrum contains the main first mode, $k=1$, with the frequencies narrowly distributed around the aver- age rotation frequency, $\langle f\rangle$; the second mode, $k=2$, with the twice wider distribution concentrated around $2\langle f\rangle$, and so on.

What distinguishes the $\mathrm{g}-2$ formula (5) from the usual Fourier transforms is that time $t_{0}$ is the same for all rotation frequencies and their modes observed at a given detector. It is the time when the center of mass of the initially very short bunch passes the detector the first time after injection. (Of course, we observe only decay electrons, not muons; however, practically, this makes no difference in the $t_{0}$-value because the signal $S(t)$ corresponds only to electrons moving with relativistic velocities, as muons do, and under small angles to the paternal muon trajectories.) It is obvious that if the bunch is sufficiently small at injection, $c \Delta t_{\mathrm{B}} \ll 2 \pi R$, and we are not interested in the analysis of the very high modes, $k \gg 1$, and, in addition, the beam line before and during injection does not cause essential variations of the muon momentum distribution along this bunch, as it is in our $\mathrm{g}-2$ 
case, then at time $t=t_{0}$ all oscillations of the fast rotation signal $F(t)$, with their different frequencies, simultaneously have maxima, as in our formula (5). Only later, when the bunch spreads longitudinally due to the revolution frequency distribution, phases of different frequencies at a given time become different. As we will see, this physical argument about initial phases is crucial for the very possibility of Fourier analysis of the muon revolution frequencies in this experiment. But once we know how to do this analysis, the Fourier transform provides very precise information about muon frequency, and hence momentum, and equilibrium radius distributions.

Formula (5) is correct not only for short bunches. If there is initially no, or almost no correlation between muon frequencies and their longitudinal phases, as in our g- 2 case, then the non-zero length of the bunch merely means the existence of some distribution of $t_{0}$ 's, $\psi\left(\Delta t_{0}\right)$, independent of the frequency distribution, $F(f)$. Taking this into account, we can average Eq. (5) over the longitudinal distribution: $\left\langle\cos \omega\left(t-t_{0}-\right.\right.$ $\left.\left.\Delta t_{0}\right)\right\rangle=\left\langle\cos \omega \Delta t_{0}\right\rangle \cos \omega\left(t-t_{0}\right)+\left\langle\sin \omega \Delta t_{0}\right\rangle$ $\sin \omega\left(t-t_{0}\right)$. If we now choose $t_{0}$ such that

$$
\left\langle\sin \omega \Delta t_{0}\right\rangle=0
$$

then, again, only the cosine Fourier term is present in Eq. (5) [4]. In the simplest case, when $\psi\left(\Delta t_{0}\right)$ is symmetric, as is likely in our case, and if $t_{0}$ is the time when the center of the (now lengthy) bunch passes a given detector the first time after injection, then Eq. (6) is true for all frequencies simultaneously, independent of distribution $F(f)$. If $\psi\left(\Delta t_{0}\right)$ is not symmetric, then according to Eq. (6), $t_{0}$ may be different for different modes $k=1,2, \ldots$. However, we analyze only the main mode, $k=1$, and since the revolution frequency distribution is very narrow, $(f-\langle f\rangle) / f \sim$ $1.4 \times 10^{-3}$, Eq. (6) gives practically the same $\Delta t_{0}$ for all frequencies in question even for a non-symmetrical bunch. For the same reason, factor $A$ in Eq. (5) is practically constant for a given mode; for the first mode, $\quad A \equiv\left\langle\cos \omega \Delta t_{0}\right\rangle \approx\left\langle\cos \left(2 \pi\langle f\rangle \Delta t_{0}\right)\right\rangle=$ constant. (Outer brackets mean averaging over the longitudinal distribution, inner brackets mean averaging over the frequency distribution.)
In Eq. (5), $F(f)$ is an actual frequency distribution unknown in advance, and not the Fourier transform, $\Phi(f)$, of the known signal $F(t)$, see formula (4) - simply because $F(t)$ is not defined at $t<t_{0}$ (in fact, at $t<t_{\mathrm{s}}, t_{\mathrm{s}}>t_{0}$ ), while the Fourier integrand must be known for the entire $(-\infty,+\infty)$ time interval. However, we can say much more than that.

Let us investigate the example of a rectangular frequency distribution in Eq. (5), the single mode, the very short bunch:

$F(\omega)=\left\{\begin{array}{cl}1 / \Omega, & \omega_{0}-\Omega / 2<\omega<\omega_{0}+\Omega / 2 \\ 0, & \text { otherwise }\end{array}\right.$

where $\omega_{0}$ is the average frequency of this distribution. Using Eq. (7), let us calculate the real and the imaginary parts of the Fourier transform of $F(t)$, $\Phi\left(\omega^{\prime}\right)=(2 / \pi) \int \mathrm{d} t F(t) \mathrm{e}^{\mathrm{i} \omega^{\prime}\left(t-t^{\prime}\right)}$ where $F(t)$ is taken from Eq. (5) and $F(f)$ from Eq. (7); $t^{\prime}$ is a constant - a very important constant, as we will see later. The (unimportant) factor $2 / \pi$ is the normalization factor. From Eqs. (5) and (7),

$$
\begin{aligned}
\operatorname{Re} \Phi\left(\omega^{\prime}\right)= & \frac{2}{\pi \Omega} \int_{\omega_{0}-\Omega / 2}^{\omega_{0}+\Omega / 2} \mathrm{~d} \omega \int_{t_{0}}^{t_{\mathrm{m}}} \mathrm{d} t \\
& \times \cos \omega^{\prime}\left(t-t^{\prime}\right) \cos \omega\left(t-t_{0}\right) \\
= & \frac{2}{\pi \Omega} \int_{\omega_{0}-\Omega / 2}^{\omega_{0}+\Omega / 2} \mathrm{~d} \omega \int_{t_{0}}^{t_{\mathrm{m}}-t_{0}} \mathrm{~d} x \\
& \times \cos \left[\omega^{\prime}\left(x+t_{0}-t^{\prime}\right)\right] \cos \omega x \\
= & \frac{1}{\pi \Omega} \int_{\omega_{0}-\Omega / 2}^{\omega_{0}+\Omega / 2} \mathrm{~d} \omega \int_{0}^{t_{\mathrm{m}}-t_{0}} \mathrm{~d} x \\
& \times\left\{\cos \left[\left(\omega+\omega^{\prime}\right) x+\omega^{\prime}\left(t_{0}-t^{\prime}\right)\right]\right. \\
& \left.+\cos \left[\left(\omega-\omega^{\prime}\right) x-\omega^{\prime}\left(t_{0}-t^{\prime}\right)\right]\right\} .
\end{aligned}
$$

The term with frequency $\left(\omega+\omega^{\prime}\right)$ in braces can be neglected since we are interested in such frequencies $\omega^{\prime}$ that $\left|\omega-\omega^{\prime}\right| \ll \omega+\omega^{\prime}$, and these two \pm combinations go to the denominators after the integration over $x$ is performed. Thus,

$$
\begin{aligned}
\operatorname{Re} \Phi\left(\omega^{\prime}\right)= & \frac{1}{\pi \Omega} \int_{\omega_{0}-\Omega / 2}^{\omega_{0}+\Omega / 2} \frac{\mathrm{d} \omega}{\omega-\omega^{\prime}} \\
& \times\left\{\sin \left[\left(\omega-\omega^{\prime}\right)\left(t_{\mathrm{m}}-t_{0}\right)-\omega^{\prime}\left(t_{0}-t^{\prime}\right)\right]\right. \\
& \left.\left.+\sin \omega^{\prime}\left(t_{0}-t^{\prime}\right)\right]\right\}
\end{aligned}
$$




$$
\begin{aligned}
= & \frac{1}{\pi \Omega} \int_{\omega_{0}-\Omega / 2}^{\omega_{0}+\Omega / 2} \frac{\mathrm{d} \omega}{\omega-\omega^{\prime}}\left\{\cos \omega^{\prime}\left(t_{0}-t^{\prime}\right)\right. \\
& \times \sin \left(\omega-\omega^{\prime}\right)\left(t_{\mathrm{m}}-t_{0}\right)-\sin \omega^{\prime}\left(t_{0}-t^{\prime}\right) \\
& \left.\times\left[\cos \left(\omega-\omega^{\prime}\right)\left(t_{\mathrm{m}}-t_{0}\right)-1\right]\right\} \\
= & \frac{1}{\pi \Omega}\left\{\cos \omega^{\prime}\left(t_{0}-t^{\prime}\right)\right. \\
\times & {\left[\operatorname{Si}\left(\omega_{0}-\omega^{\prime}+\Omega / 2\right)\left(t_{\mathrm{m}}-t_{0}\right)\right.} \\
& \left.-\operatorname{Si}\left(\omega_{0}-\omega^{\prime}-\Omega / 2\right)\left(t_{\mathrm{m}}-t_{0}\right)\right]-\sin \omega^{\prime}\left(t_{0}-t^{\prime}\right) \\
& \times\left[\operatorname{Ci}\left(\omega_{0}-\omega^{\prime}+\frac{\Omega}{2}\right)\left(t_{\mathrm{m}}-t_{0}\right)-\operatorname{Ci}\left(\omega_{0}-\omega^{\prime}-\frac{\Omega}{2}\right)\right. \\
& \left.\left.\times\left(t_{\mathrm{m}}-t_{0}\right)-\ln \frac{\omega_{0}-\omega^{\prime}+\Omega / 2}{\omega_{0}-\omega^{\prime}-\Omega / 2}\right]\right\} .
\end{aligned}
$$

Here $\operatorname{Si}(z), \operatorname{Ci}(z)$ are sine and cosine integrals. They are tabulated for positive $z$, but Eq. (9.3) is correct for both positive and negative values of $\left(\omega-\omega^{\prime}+\Omega / 2\right)$ and $\left(\omega-\omega^{\prime}-\Omega / 2\right)$, taking into account the following properties of $\mathrm{Si}(z), \mathrm{Ci}(z)$ : $\operatorname{Si}(-z)=-\operatorname{Si}(z), z \geqslant 0 ; \operatorname{Ci}(-z)=\operatorname{Ci}(z)-\mathrm{i} \pi, z>0$; $\mathrm{Ci}(-z)-\ln (-z)=\mathrm{Ci}(z)-\ln (z), \quad z>0 ; \mathrm{Ci}(z)-$ $\ln (z) \rightarrow 0.5772 \quad$ when $\quad z \rightarrow 0 . \quad \operatorname{Si}(z) \rightarrow \pi / 2-$ $\cos (z) / z, \operatorname{Ci}(z) \rightarrow \sin (z) / z$, when $z \rightarrow \infty$,

$z=\left(\omega_{0}-\omega^{\prime} \pm \Omega / 2\right)\left(t_{\mathrm{m}}-t_{0}\right)$.

The plus or minus sign in Eq. (10) corresponds to the signs in Eq. (9.3). We assume a sufficiently long duration $\left(t_{\mathrm{m}}-t_{0}\right)$ of the observed signal, so $z \gg 1$ for practically all values of $\omega^{\prime}$, with the exclusion of only two areas around the sharp borders of the frequency distribution in this example, where $\omega^{\prime}=$ $\omega_{0} \pm \Omega / 2$. Note that when $\omega^{\prime}$ is inside our frequency distribution, the two sine integrals in Eq. (9.3) have different signs and are summarized, and when $\omega^{\prime}$ is outside of that distribution they have the same signs and hence cancel each other out. Taking this into account and neglecting terms of the order $1 / z, z \rightarrow \infty$, we therefore have the following approximate formula for $\omega^{\prime} \neq \omega_{0} \pm \Omega / 2$ :

$$
\begin{aligned}
\operatorname{Re} \Phi\left(\omega^{\prime}\right)= & F\left(\omega^{\prime}\right) \cos \omega^{\prime}\left(t_{0}-t^{\prime}\right) \\
& +\frac{\sin \omega^{\prime}\left(t_{0}-t^{\prime}\right)}{\pi \Omega} \ln \left|\frac{\omega_{0}-\omega^{\prime}+\Omega / 2}{\omega_{0}-\omega^{\prime}-\Omega / 2}\right|
\end{aligned}
$$

Similar calculations lead to the approximate formula for the imaginary part:

$$
\begin{aligned}
\operatorname{Im} \Phi\left(\omega^{\prime}\right)= & F\left(\omega^{\prime}\right) \sin \omega^{\prime}\left(t_{0}-t^{\prime}\right) \\
& +\frac{\cos \omega^{\prime}\left(t_{0}-t^{\prime}\right)}{\pi \Omega} \ln \left|\frac{\omega_{0}-\omega^{\prime}+\Omega / 2}{\omega_{0}-\omega^{\prime}-\Omega / 2}\right| .
\end{aligned}
$$

These formulas for the rectangular frequency distribution (7) expose the "anatomy" of the distortion, which appeared to be logarithmic, of the actual frequency distribution by the Fourier transform of the half-time signal. But we can see also that it is possible to extract the correct frequency distribution from these formulas in the following three cases.

(a, b) $\Omega \rightarrow 0, \Omega \rightarrow \infty$. In these cases the logarithmic terms in Eqs. (11) and (12) go to zero, and since $F_{\max }(\omega) \sim 1 / \Omega$, the factors before the algorithms in formulas (11) and (12) have the same order as $F\left(\omega^{\prime}\right)$, so when the logarithms go to zero, the second terms in Eqs. (11) and (12) can be neglected. Therefore, the usual Fourier transform leads to the correct result, $\Phi(\omega)=F(\omega)$. However, we are not interested in these two cases.

(c) $t^{\prime}=t_{0}$, the case in which we use a very special Fourier transform, namely the cosine transform with the time counted from the initial time $t_{0}$, i.e., from the moment when the center of the muon bunch passes the detector immediately after injection. In this case,

$\operatorname{Re} \Phi(\omega)=F(\omega), \quad t^{\prime}=t_{0}$.

Fig. 3a shows the result of a simulation in the case when a very short bunch injected into the g-2 ring has the frequency distribution (7), time $t_{0}$ of the initial passing the given detector is known, and $F(t)$ can be (and has been) calculated directly using beam dynamics. After that we have applied the cosine Fourier transform to $F(t)$ from the starting time $t_{\mathrm{s}}$ such that $t_{\mathrm{s}}=t_{0}=0$. The result is not ideal only because we analyze a (non-realistic) rectangular distribution function disconnected at two frequencies. In Fig. 3b, the starting time is $10 \mathrm{~ns} \neq t_{0}=0$. We see that even if this time is much smaller than our revolution period, $T=$ $149 \mathrm{~ns}$, the distortion of the actual distribution is tremendous. (In our 1999 measurement of the 

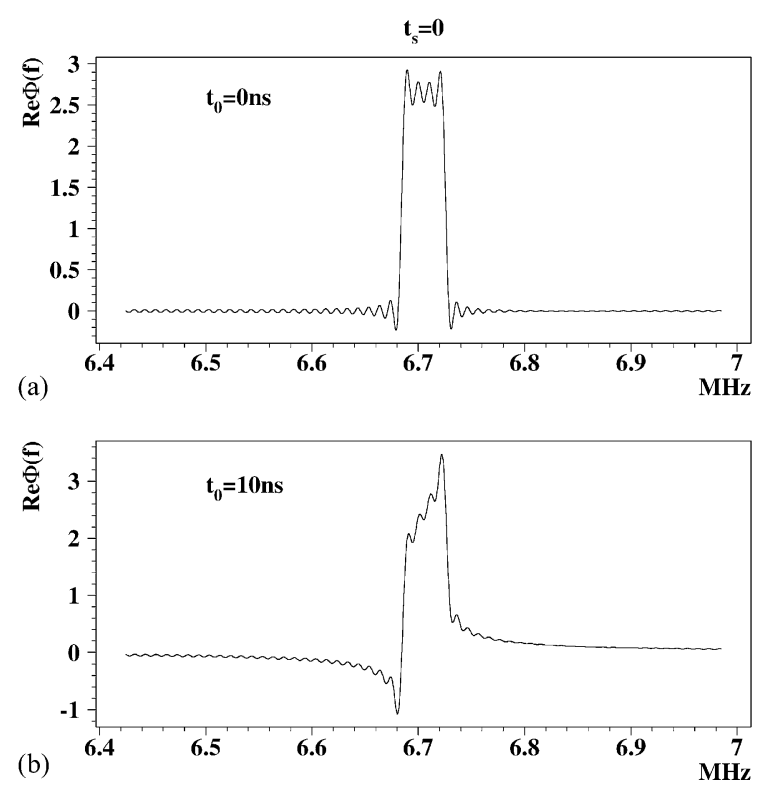

Fig. 3. Cosine Fourier image of the rectangular distribution, zero starting time $t_{\mathrm{s}}$ (simulations); (a) correct initial time $t_{0}$; (b) incorrect initial time.

muon frequency distribution, the achieved accuracy of the initial time value was $\pm 0.5 \mathrm{~ns}$, see the next section.)

Rule (13), see also Eq. (6), was first theoretically found in Ref. [4] for an arbitrary frequency distribution, $F(\omega)$, and was confirmed by simulations which assumed that the initial time, $t_{0}$, was known and that the fast rotation signal could be observed beginning from that very time, $t_{\mathrm{s}}=t_{0}$. The last assumption appeared to be unrealistic for our g-2 experiment because signals can be observed only later, $t_{\mathrm{s}}>t_{0}$. The needed corrections are described in the next section.

\section{Analysis of the fast rotation signal known only at later times}

The g-2 and hence the fast rotation signal can be observed at a given detector only after its gate is open, that is, some several microseconds after injection. In connection with this, there are two problems in analyzing the muon frequency distribution. First, since initial time $t_{0}$ is not directly measured, an iteration procedure for determining it with the needed accuracy must be found. Second, the iteration procedure for extracting the frequency distribution from the data beginning not from $t=t_{0}$, but from some later time, $t_{\mathrm{s}}>t_{0}$, must also be found. It seems obvious that the smaller the $t_{\mathrm{s}}$, the easier the analysis. (In the 1999 run, the smallest possible starting time, $t_{\mathrm{s}}$, for the observation of the fast rotations was $4.7 \mu \mathrm{s}$, some 32 turns after injection.)

The first task is to determine $t_{0}$ in the first approximation. For this we must work with the fast rotation signal, $F(t)$, see formula (4), where $S(t)$ in the numerator is the actual g-2 signal (in the chosen detector). The denominator is either the five parameter fit for this detector, as in our case, or a more accurate fit function. Of course, the fast rotation itself must not be included in the fit function in the denominator. Note that besides frequencies around the main first mode (the usual revolution frequencies), $F(t)$ contains information about higher modes, $k f, k=2,3, \ldots$. However, one can see from Fig. 2 that after $t \sim 30 \mu$ s (about 200 turns), the fast rotation signal $F(t)$ becomes equal to the sum of a constant part of the muon current and oscillations having only one frequency, namely, the average revolution frequency, $\langle f\rangle$, the amplitude of which is slowly damping, plus some fluctuations. The physical cause of the presence of only one visible frequency at late times is the phase mixing of oscillations having different frequencies inside the same mode, with the phases of the highest modes, $k>1$, mixing much faster than the phases of the frequencies distributed around the main first mode, $k=1$. As a result, the highest modes disappear in the time-domain signal $F(t)$ much faster than the main mode and, in the main mode, the average frequency $\langle f\rangle$ dominates. (Note that in the frequency domain, none of the frequencies disappear; frequency distribution $F(f)$ is an integral of motion in the g-2 ring.) In fact, we can well distinguish the average frequency $\langle f\rangle$ of the main mode in $F(t)$ much earlier, several microseconds after injection.

Now, using the existence of the clearly visible one-frequency oscillations, we define frequency $\langle f\rangle$ in the first approximation directly from $F(t)$, and then count an integer number of the average 
revolution periods, $\langle T\rangle$, backwards in time, beginning from some good maximum of $F(t)$, down to the very first turn after injection. This way we obtain the initial time, $t_{0}$, in the first approximation. Its more precise value must be defined by iterations later. Though we find $t_{0}$ only for one frequency, a physical argument described above is that all other revolution frequencies in question have simultaneous maxima at the same time $t_{0}$ (for a given detector) and, therefore, every frequency is present in the signal in the form $F(f) \cos \omega\left(t-t_{0}\right)$, with the same initial time $t_{0}$.

Our next step is to calculate the cosine Fourier integral using the data available for a given detector and the first approximation for the initial time for this detector:

$\operatorname{Re} \Phi\left(f, t_{\mathrm{s}} ; t_{\mathrm{m}}\right)=\int_{t_{\mathrm{s}}}^{t_{\mathrm{m}}} F(t) \cos 2 \pi f\left(t-t_{0}\right) \mathrm{d} t$

for those revolution frequencies, $f$, first mode, which are accepted by the g- 2 ring, and some $f$ 's beyond that area for a purpose to be explained later. (It is possible and interesting also to analyze the higher modes, $k>1$, but we will not touch on this subject here.) To have a better iteration process, starting time $t_{\mathrm{s}}$ should be taken at some maximum of the function $F(t)$ after the gate-on time of the chosen detector. As for $t_{\mathrm{m}}$, we have shown in the previous section using our example that, for a sufficiently big upper time, integral (14) does not depend on it. In accordance with the results of many simulations, the sufficiently big upper limit in the g-2 experiment should be above 250-300 $\mu$ s. Having this in mind, from now on we will omit $t_{\mathrm{m}}$ from $\Phi\left(f, t_{\mathrm{s}} ; t_{\mathrm{m}}\right)$ writing $\Phi\left(f, t_{\mathrm{s}}\right)$ instead. (This function depends also on $t_{0}$, but we can omit $t_{0}$ because there is only one correct value of it.)

The theory and simulations prove that if $t_{\mathrm{s}}=t_{0}$ and $t_{0}$ is found correctly, and statistics is not limited, $\operatorname{Re} \Phi\left(f, t_{0}\right)$ is exactly proportional to the actual muon revolution frequency distribution $F(f)$, if this distribution has any physically reasonable shape. However, the fast rotation signal $F(t)$ is known only for times $t>t_{\mathrm{s}}>t_{0}$. Moreover, up to now we know how to find $t_{0}$ only in the first approximation. In order to understand how to refine $t_{0}$, look first at Fig. 4 which shows

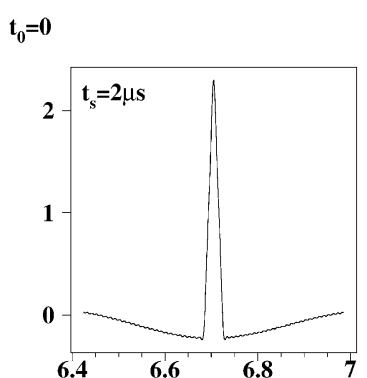

(a)
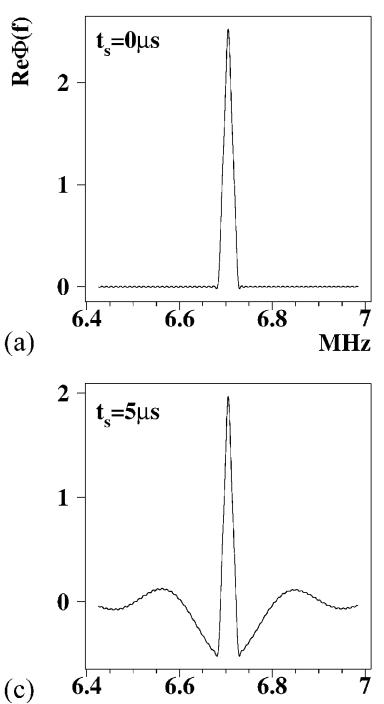

(b)

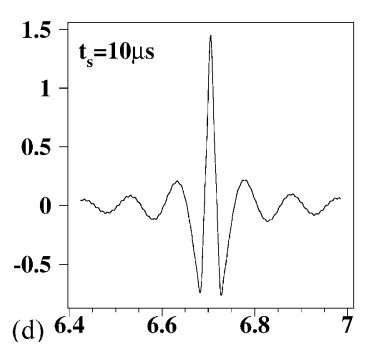

Fig. 4. Cosine Fourier image of the triangle distribution, correct initial time $t_{0}$ (simulations): (a) zero starting time $t_{\mathrm{s}}$; (b)-(d) non-zero starting times.

$\operatorname{Re} \Phi\left(f, t_{\mathrm{s}}\right)$ for different $t_{\mathrm{s}}$ 's. These are results of simulations of the fast rotation signal, in which $t_{0}$ is assumed to be known. In this simulation, the envelope of the muon revolution frequency distribution $F(f)$ is taken as a symmetric triangle. (In reality, our $F(f)$ appears to be only slightly asymmetric, see Fig. 1; such small asymmetry does not increase the number of iterations needed for our accuracy.) In Fig. 4a, we see that the frequency distribution is reconstructed precisely by integral (14) when $t_{\mathrm{s}}=t_{0}$, as it must be. But it is not reconstructed precisely when $t_{\mathrm{s}} \neq t_{0}$; there is a background shown in Figs. $4 \mathrm{~b}-\mathrm{d}$. The bigger the $t_{\mathrm{s}}$ is, the bigger and more complicated the background is around the actual frequency distribution. But the shape of the central part of the distribution is not disturbed. We can use this central part as the first approximation for $F(f)$. Now look at Figs. $5 \mathrm{a}-\mathrm{d}$. These are also the results of the simulations with the same frequency distribution, but with erroneous $t_{0}$ 's, with different deviations of $t_{0}$ in integral (14) from the correct initial time $t_{0}=0$. Starting time $t_{\mathrm{s}}$ is the same in all cases, $\left(t_{\mathrm{s}}-t_{0}\right)=5 \mu \mathrm{s}$. We see that even when the deviation of $t_{0}$ from the correct initial time equals 
(a)
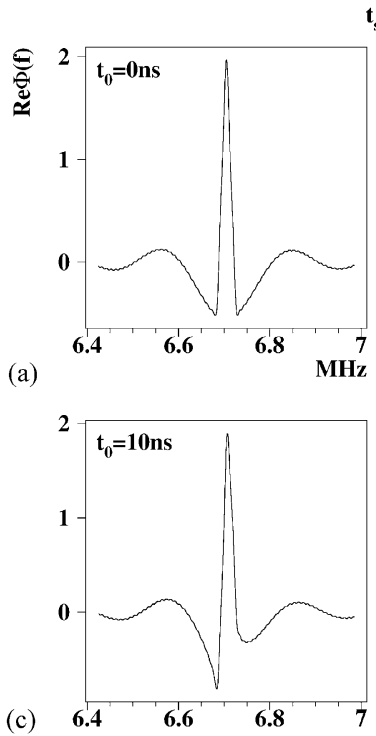

$t_{\mathrm{s}}=5 \mu \mathrm{s}$

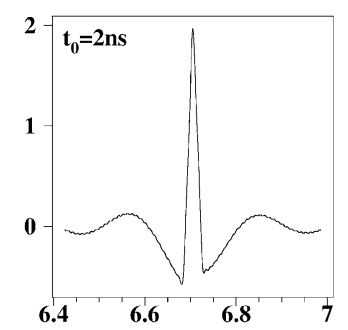

(b)

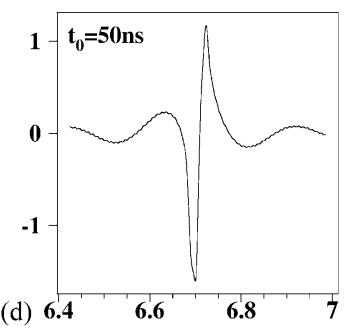

Fig. 5. Cosine Fourier image of the triangle distribution, nonzero starting time (simulations): (a) correct initial time; (b)-(d) incorrect initial times.

only $2 \mathrm{~ns}$, the central part of the image becomes visibly different from the original. This means that $t_{0}$ must be found with accuracy $\sim 0.5 \mathrm{~ns}$ (some 0.003 of the revolution period).

Simultaneously, with the central part, the background changes when $t_{0}$ is changed; however, further analysis shows that in the nearest neighborhood of $\langle f\rangle$, the background must be a symmetric parabola, independent of the actual frequency distribution, see formula (19) and Fig. 6c. Taking this into account, we conclude with certainty that a very good second approximation is a $t_{0}$ that corresponds to the most symmetric background of $\operatorname{Re} \Phi\left(f, t_{\mathrm{s}}\right)$ (calculated in accordance with Eq. (14)) outside the borders of the vacuum chamber marked by vertical lines in Fig. 6 . It appears that this second approximation is sufficient for the needed accuracy.

Now, when we know the initial time we can get the muon revolution frequency distribution $F(f)$. The idea is the following. Although the fast rotation signal $F(t)$ is unknown at times $t_{0}<t<t_{\mathrm{s}}$, it objectively exists. If $F(t)$ for all times $t>t_{0}$ and the initial time itself were known exactly, then - for physical reasons explained above - the
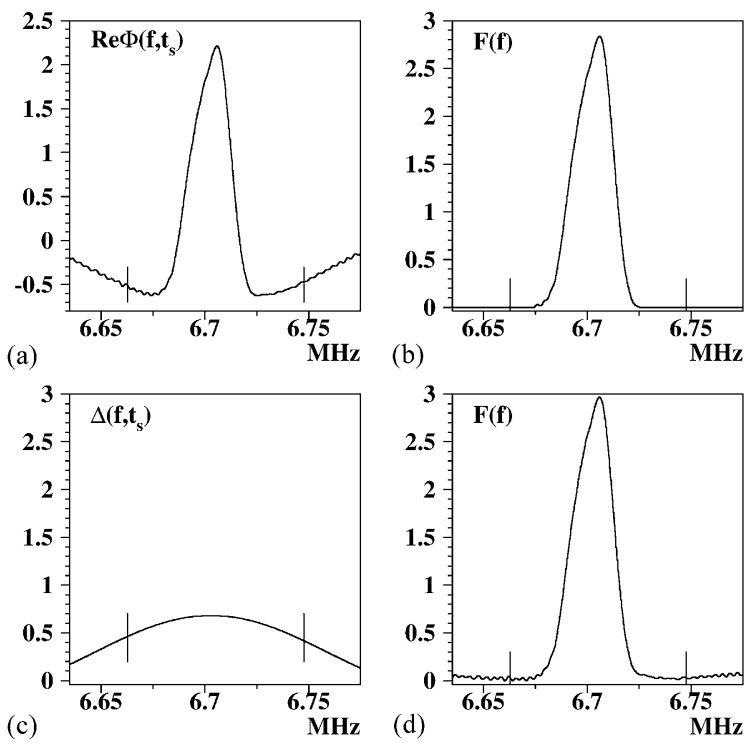

Fig. 6. Four-step procedure to get the correct partial time Fourier image (g-2 experiment). (a) Cosine Fourier image of the fast rotation signal, non-zero starting time. The initial time is known in the first approximation. (b) The first approximation of the revolution frequency distribution. (c) The non-observed part of the cosine Fourier image. (d) The second approximation of the revolution frequency distribution.

muon revolution frequency distribution, $F(f)$, could be found as follows (see (14)):

$\int_{t_{0}}^{t_{\mathrm{m}}} F(t) \cos 2 \pi f\left(t-t_{0}\right) \mathrm{d} t=A F(f)$

where $A$ is a constant depending on the initial azimuthal distribution of muons, as explained above. However, even if we know $t_{0}$, we still do not know the following small part $\Delta\left(f, t_{\mathrm{s}}\right)$ of integral (15):

$\Delta\left(f, t_{\mathrm{s}}\right)=\int_{t_{0}}^{t_{\mathrm{s}}} F(t) \cos 2 \pi f\left(t-t_{0}\right) \mathrm{d} t$

$\operatorname{Re} \Phi\left(f, t_{\mathrm{s}}\right)+\Delta\left(f, t_{\mathrm{s}}\right)=A F(f)$.

But we can calculate $\Delta\left(f, t_{\mathrm{s}}\right)$ in the first approximation, which appears to be a final approximation for our measurement, simply by using the central part of the $\operatorname{Re} \Phi\left(f, t_{\mathrm{s}}\right)$. This is possible because the background described by the function $\left(-\Delta\left(f, t_{\mathrm{s}}\right)\right)$ is almost constant in the central part of the distribution. 
An example of the procedure actually used in our g-2 experiment is illustrated in Fig. 6. By calculating integral (14) with different $t_{0}$ 's we found the best initial time, which corresponds to Fig. 6a with a symmetric background. The central part of Fig. $6 a$ gave us the main mode of the frequency distribution in the first approximation, Fig. 6b. Using this, we calculated Eq. (16) in the form (see (5)):

$\Delta\left(f, t_{\mathrm{s}}\right)=a \int \mathrm{d} f^{\prime} F\left(f^{\prime}\right) \frac{\sin 2 \pi\left(f-f^{\prime}\right)\left(t_{\mathrm{s}}-t_{0}\right)}{f-f^{\prime}}+b$.

Constant $a$ is added to not bother about normalization (which is not taken into account in Eq. (16) either). Constant $b$ takes into account that the bottom of the frequency distribution, an approximate part of which is represented by the center of the curve $6 \mathrm{a}$, is not exactly defined there. Integral (18) with some arbitrary $a$ and $b$ and $F(f)$ from Fig. 6b, is shown in Fig. 6c. It is almost an exact parabola, as it must be since, for small $(f-\langle f\rangle)\left(t_{\mathrm{s}}-t_{0}\right)$

$$
\begin{aligned}
& \Delta\left(f, t_{\mathrm{s}}\right)=b+a\left(t_{\mathrm{s}}-t_{0}\right) \\
& \times\left\{1-\frac{2 \pi^{2}\left(t_{\mathrm{s}}-t_{0}\right)^{2}}{3}\left[(f-\langle f\rangle)^{2}+\sigma^{2}\right]+\cdots\right\}
\end{aligned}
$$

$$
\langle f\rangle=\int f \mathrm{~d} f F(f), \quad \sigma^{2}=\int f^{2} \mathrm{~d} f F(f)-\langle f\rangle^{2} .
$$

Finally, we have chosen $a$ and $b$ such that, for the frequencies outside the area permitted by the vacuum chamber (the borders of which are marked by the vertical lines in Fig. 6), the left part of Eq. (17), and hence $F(f)$, equals zero. The obvious reason is that there are no muons beyond those borders. This gives the next (and final) approximation, for the needed accuracy, of the frequency distribution shown in Fig. 6d.

\section{References}

[1] H.W. Brown, et al., g-2 Collaboration, Phys. Rev. Lett. 85 (2001) 2227.

[2] D.H. Brown, et al., BNL AGS E821. A New Precision Measurement of the Muon (g-2) Value at the Level of 0.35 ppm, 3rd Edition, March 1995 (design report), B.L. Roberts (Ed.), unpublished.

[3] J. Bailey, et al., Nucl. Phys. B 150 (1979) 1.

[4] Y.F. Orlov, A. Soffer, New method of muon momentum distribution measurement in (g-2) experiment, in: $\mathrm{T}$. Hasegawa, et al. (Eds.), Frontiers of High Energy Spin Physics, Proceedings of the 10th International Symposium on High Energy Spin Physics, Nagoya, Nov. 9-14, 1992, Universal Academy Press, Tokyo, 1993, p. 723. 